\title{
Sustainable Services: The Natural Mimicry Approach
}

\author{
Adi Wolfson ${ }^{1}$, Dorith Tavor ${ }^{1}$, Shlomo Mark ${ }^{2}$ \\ ${ }^{1}$ Green Processes Center, Sami Shamoon College of Engineering, Beer Sheva, Israel; ${ }^{2}$ Negev Monte Carlo Research Center, Sami \\ Shamoon College of Engineering, Beer Sheva, Israel. \\ Email: adiw@sce.ac.il
}

Received January $18^{\text {th }}, 2011$; revised March $6^{\text {th }}, 2011$; accepted April $13^{\text {th }}, 2011$.

\begin{abstract}
Environmental issues have become an integral factor in any decision-making process and in the design and implementation of manufacturing and agricultural production systems. As the service sector continuously grows in size and importance and service systems become more comprehensive, complex, and interdisciplinary, the creation of new services should be imbued with sustainability as the primary value of each service and as a service in and of itself. The paper presents a natural mimetic approach that follows the ground rules of nature to characterize the sustainability of a service and to choose the most sustainable service alternative.
\end{abstract}

Keywords: Service Science, Service System, Sustainability, Lifecycle, Service Lifecycle, Sustainable Service, Nature Mimic

\section{Introduction}

The service sector is continuously growing in size and importance, and service systems are becoming more comprehensive, complex, and interdisciplinary [1-3]. This fact was one of the primary incentives behind establishing a new track for service research, i.e., service science, the study of service systems value co-creation of people, information, and technology (e.g., language, laws, measures, and methods) [4,5] to create a basis for systematic service innovation.

Service is traditionally defined as whatever is not agriculture or manufacturing [6-8] and that which involves a transformation of values and methods by the service provider into a product (i.e., service) requested by the customer. However, because the service sector integrates different disciplines, such as marketing, computer science, information systems, and operations, service philosophy has in recent years shifted from service products that are economically measurable and management-oriented to values that are more operational and socially responsible.

Environmental issues have become an integral factor in any decision-making process and in the design and implementation of manufacturing and agricultural production systems. Sustainability, defined as the capacity of an ecosystem to bear the stress of processes while maintaining them into the future and preserving enough space for subsequent generations, is currently the leading paradigm $[9,10]$. It integrates economic, social, and environmental elements into different measures and indicators, ecological and carbon footprints among them. Recently, the concept of life cycle assessment (LCA), which is an analysis of the sum of materials and energy involved in the production, distribution, use, and disposal steps of a tangible product, was also adopted to optimize the environmental performance of processes [11,12].

Similarly, a new service approach, e.g., sustainable service, based both on the rational use of natural resources and on acting with environmental awareness, was recently introduced $[13,14]$. Sustainable service also facilitates conceptualizing services as shared and dynamic problem-solving endeavors that create value in multiple dimensions, and it takes into account potential long-term effects. The development of sustainable services also requires a life cycle-oriented perspective on the relationships between consumers, providers, and suppliers [15] and a desire to be ecosystem compatible. Yet, the fundamentals of sustainable service and strategies for making a service more sustainable are still in development.

We recently presented a novel perspective together with a model that describes the relationship between sus- 
tainability and service within the framework of service science [16]. The new perspective defines sustainable service not just as a service that fulfills customer demands and that can be perpetuated for long periods of time without having a negative impact on either the natural or the social environment, but also as a basic value and as an essential part of each service, and in fact, as a "superservice" in and of itself. More specifically, the new model describes sustainable service as the integration of tangible and intangible resources together with manufacturing and agriculture. Service customers comprising the current generation simultaneously evolve into active suppliers who deliver sustainable values to the next generation, as illustrated in the " $\mathrm{S}^{3}$ Sustainability as Service Science" Model (Figure 1). Additionally, sustainable service should also generate new and alternative values for supplying customer demands more sustainable.

The model comprises two main stages (Figure 1) first, a sustainable decision made by a service, with said decision relying on the service's resources, including natural resources, technologies, and information and knowledge; second, the most sustainable choice is selected from among the alternatives after evaluating each in terms of its integration of services and of manufacturing and agricultural processes.

In addition, because sustainability represents the combined influences of environmental, economic, and social elements, sustainability values which will represent the full sustainability image of a process based on a comparative scale such as its carbon footprint, an assessment of its impact on health, or its gross domestic product should be used [16].

\section{Nature's Ground Rules}

Current global realities make it imperative to move in the direction of sustainable services. Herein, we demonstrate an innovative approach to characterizing sustainable services will facilitate the decision making process when choosing between different service alternatives based on nature's ground rules.

From the very beginning, nature has inspired and challenged scientists. Biosystems present an excellent example of natural, efficient, green, low energy, and compatible, multistep processes that function in concert. The need to develop more efficient and cleaner ("greener") production systems and services is a universal concern. Nature itself provides many services to promote and sustain ecosystems and benefit humans, such as air cleansing and temperature control by trees and water filtration by swamps [17].

The primary driving force behind nature, and in fact, its main role, is the perpetuation of life, which is accomplished through smart and efficient life cycles that function together in concert with a future-oriented perspective, adapting to changes over time. Human efforts at developing sustainable services can benefit from nature's model by adopting a natural mimicry approach that entails making these fundamental ideas inherent to every sustainable service.

\section{Sustaining Life-Future-Oriented Service}

Because nature's main incentive is the perpetuation of life, each natural process or service is future-oriented. Likewise, the core of sustainability entails living comfortably but responsibly today, which necessarily implies preserving the rights of coming generations to live equally well. In this respect, sustainable service should be imbued with future-oriented thinking to maintain global sustainability, which should be a primary value of each and every service. But at the same time, as services and production processes are usually tied together, sustainability should also participate in a super-service that in-

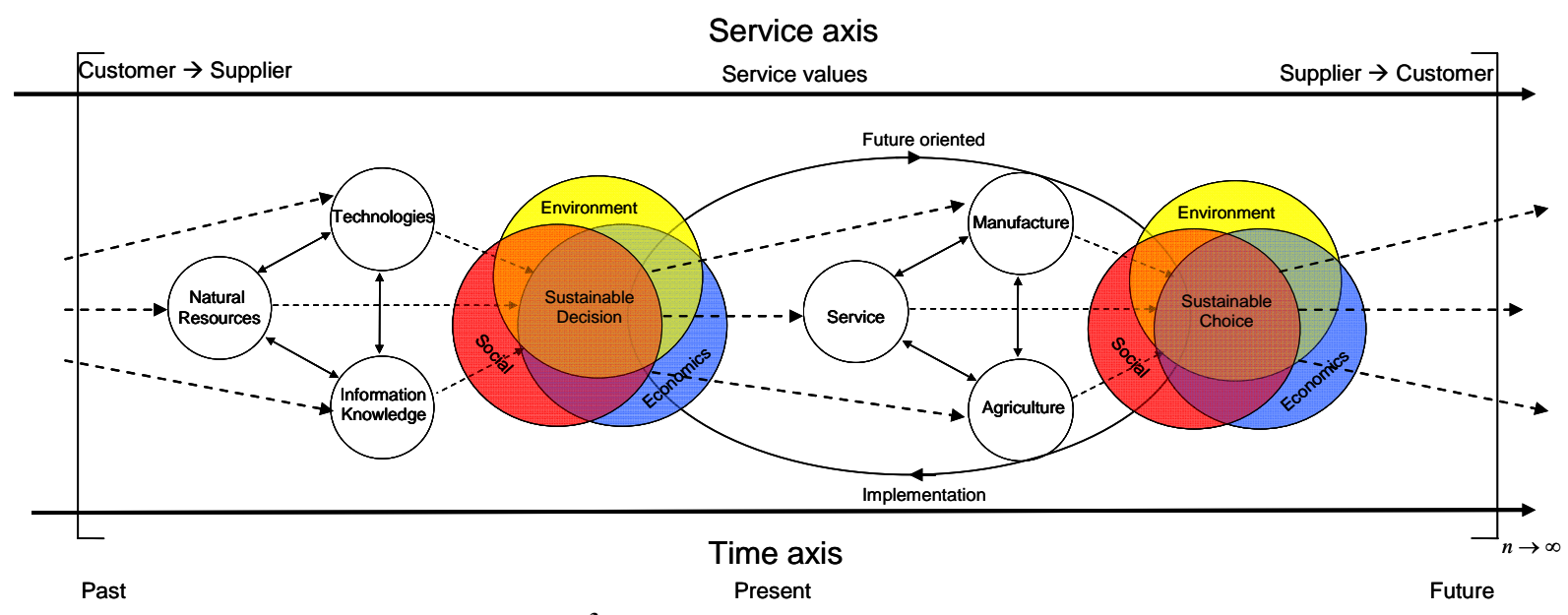

Figure 1. $\mathrm{S}^{3}$-Sustainability as Service Science Model. 
cludes services and manufacturing and agricultural processes, and customers who comprise the current generation simultaneously evolve into active suppliers who deliver sustainable values to the next generation (Figure 2).

\section{Energy-Service Quantification}

Systems can be quantified in terms of energy, the concept of which, and its transformation, are useful in explaining and predicting most natural phenomena. However, beyond the physical definition of energy, it is also accepted as a measure of life. In general, each natural process is based on the minimum use of material energy to sustain life. In this respect, to survive, animals and plants developed efficient ways to live and reproduce, while using minimum resources.

The driving-force of every process is a potential gradient such as a pressure gradient that causes fluid motion or a temperature gradient that leads to heat transfer that can be translated into a change in energy. Similarly, each service is based on a gradient of need between the customer and the provider. The customer needs what the provider is selling. This driving force produces a value that is transferred between the two parties. However, besides a need gradient, sustainable service should also be driven by sustainable decision making that asks whether the service is necessary, uses a future-oriented viewpoint, and considers existing natural resources, technologies, and knowledge and information to ensure that global sustainability will be preserved.

It should be noted that although services are intangible products that do not directly use energy and materials, they are based on natural resources and different technologies and manpower that constantly use materials and energy for their operation. Hence, sustainable service should also be based, akin to natural processes, both on the minimum use of energy and materials and also on efficiency, which should be calculated from the service supply chain and technologies in use all the way to customer operations. Therefore, service sustainability compels implementation of a service that employs sustainable supplies and that uses clean technologies while encouraging the customer to take responsibility for its actions and to supply sustainability to the next generation.

Finally, as defined by the basic laws of physics, the conservation of mass and energy are fundamental to life, and neither can be created nor destroyed. In other words, the energy and materials balance of a sustainable service must ensure that the resources invested by the provider will be eventually delivered by the customer to the coming generations for its re-use.

\section{Entropy-Service Quality}

Although the transfer and balance of mass and energy are vital to understanding each process, more detail is needed to describe natural systems and life. The direction and efficiency which the energy is transferred is crucial in determining whether a process will be carried out. In other words, although a temperature gradient is the driving force for heat transfer, the spontaneous transfer of heat is possible in only one direction, from high to low temperature. Moreover, a certain amount of heat is lost in its conversion to work. These two phenomena are defined in the second law of thermodynamics, which deals not only with the quantity of energy, but also with its quality [18]. The second law defines a property intrinsic to each system, named entropy, which dictates the direction of change that occurs in a system during a process. As such, a system naturally progresses in the direction of increasing entropy, and the smaller the change in entropy undergone by a system during a process, the greater the efficiency of that process. Alternatively, in statistical mechanics, entropy is a measure of the number of ways

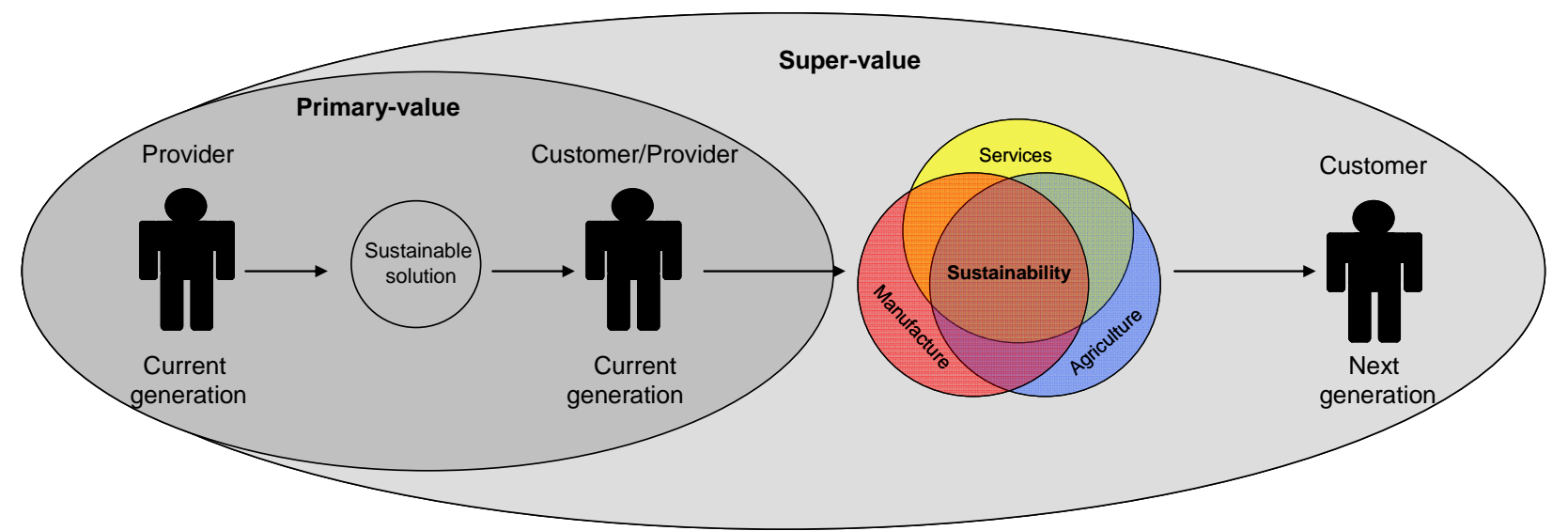

Figure 2. Future-oriented sustainable service. 
in which a system can be arranged, often termed the "disorder" of a system, such that increased entropy signifies greater disorder.

To minimize the impact a service has on mass and energy, we should define sustainability as a state of a service and measure the change in sustainability during implementation of the service $(\Delta \mathrm{S})$. The $\Delta \mathrm{S}$ should be calculated from the various sustainability indicators (mentioned above) and integrated into a single-scale number that facilitates the decision making process as to whether a service is sustainable, which, in turn, contributes to making a sustainable decision (Figure 1). In general, as with entropy, every process, including a service, utilizes materials and energy and thus increases the sustainability $(\Delta \mathrm{S}>0)$, while the lower the $\Delta \mathrm{S}$ is the more sustainable the service/process is $(\Delta \mathrm{S} \rightarrow 0)$. However, like energy, which can neither be created nor destroyed (the first law of thermodynamics), the selection of a sustainable service should also consider its integration and balance with other services and with manufacturing and agricultural processes, with the goal being to minimize the overall sustainable change (Overall $\Delta \mathrm{S}_{\min }$ ). An evaluation of overall sustainable change, therefore, will enable the selection of the most sustainable choice from among the alternatives (Figure 1).

Moreover, just as entropy is a measure of the number of ways in which a system can be arranged, there are usually a variety of routes for delivering a given service. Thus, the entropy of a sustainable service is also represented by two functions: a decision about whether the service in and of itself is sustainable, i.e., a sustainable decision, and then the selection of the most sustainable service from among the alternatives and its integration with other processes to yield the most sustainable choice $(\Delta \mathrm{S} \rightarrow 0)$.

\section{Life Cycle-Service Renewability}

Originally applied in biology to describe the generational progression of a species via its reproduction, the term life cycle is future oriented insofar as it entails using minimum energy for maximum efficiency. For the same reasons, nature works in cyclic processes - the carbon cycle, the water cycle, etc.- to balance and regulate earth and its atmosphere.

The concept of life cycle or cyclic process, however, is not limited in its applicability to natural processes; it is also used widely in the economics and management fields and even to characterize services. The life cycle of a tangible product, for example, covers the product from the design and development process to its launch into the market until eventually the market stabilizes and the product matures. Eventually, it is overtaken by its competitors and it goes into decline, after which it is ulti- mately withdrawn from the market.

In addition to the commercial-oriented life cycle of a product, there is also the material-oriented life cycle, which traces the utilization of materials from when they exit the production process to their use and disposal until they are finally recycled or recovered. Recycling, the directed exploitation of materials from one product to create another to prevent waste is an example of incorporating a life cycle perspective into sustainable life. Alternatively, the production of new, biodegradable products that can be recovered after their use, such as corn based polymers, is today expanding with its wider acceptance as a more sustainable production route.

Nevertheless, besides tracking the phases of a process or a product and the materials it uses, a life cycle approach also allows for system analysis and for measuring the system's environmental, social, or economical impact on life, i.e., life cycle analysis or assessment. A life cycle assessment entails a method to determine the influence of all the stages of a product or process, from "cradle-tograve," on the environment, taking into account material and energy consumption from the point of development through manufacturing to marketing, distribution, and disposal or recycling. It is in fact a measure of all the resources required in the overall life cycle of a product or a process [11,12].

As such, life cycle can be used to describe the stages of a service, from its planning and design to its delivery and eventual use. Yet, applying the concepts of life cycle to sustainable service should also take into account the rational use of resources, knowledge and information, and clean technologies while aspiring to maximum efficiency and the integration of the service with other services and agricultural and manufacturing processes in a future oriented perspective. These components should be integrated in the different phases of a service, from its design and development to its management and implementation. Therefore, the life cycle of a sustainable service should integrate the life cycles of each component of the new "super-service" and consider both tangible and intangible resources. Finally, to complete the cycle, a new value is created that transfers sustainability from the customer, who now becomes a supplier, to the next generations, i.e., it is a renewable service (Figure 3).

\section{Evolution-Spiral Service}

Finally, from an evolutionary viewpoint, life is a process in which each population adapts to various changes and goes through various selection steps. In the same manner, tangible and intangible products have to track changes in resources, technologies, and information and knowledge and incorporate those changes into their life cycles. Moreover, a sustainable service should be comprehensive, 


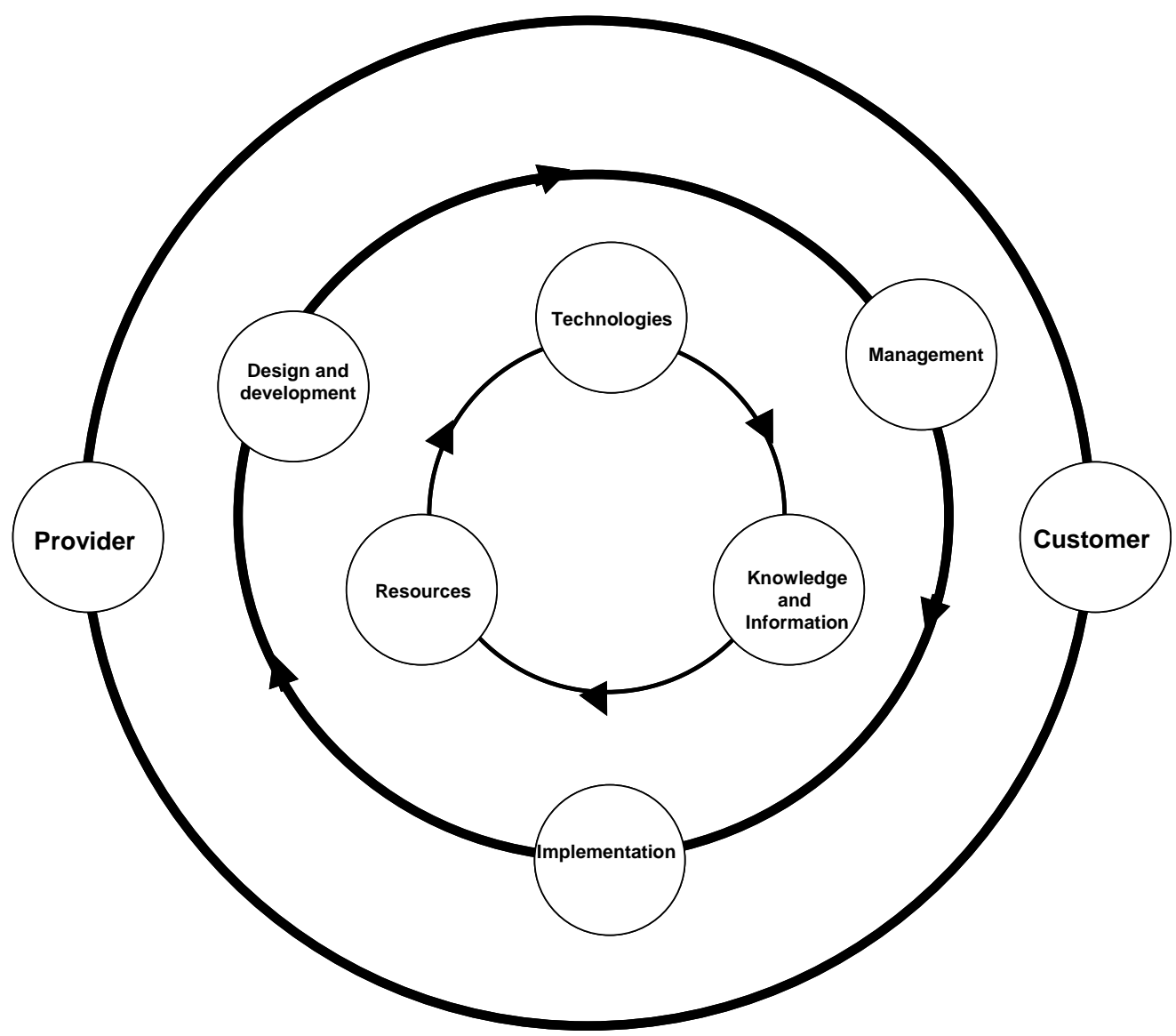

Figure 3. Sustainable service life cycle.

integrative, and smart, such that it actively anticipates future scenarios (e.g., availability of resources, new competition, etc.) instead of simply reacting to change as it happens. In other words, a sustainable service can exist as a spiral, multi-dimension "super-service" that transfers sustainability over time while constantly learning and adapting to changes in suppliers, customers, and values $\left(\lim _{\mathrm{t} \rightarrow \infty} \Delta \mathrm{S}=0\right)$.

\section{Summary}

Sustainable service is that which fulfills customer demands and that can be continued for long periods of time without having a negative impact on either the natural or the social environment. Furthermore, it is imbued with sustainability as its primary value and as a "super-service" in and of itself. Herein, an innovative approach that uses nature's ground rules to characterize sustainable services and choose between different service alternatives was demonstrated. It was suggested that sustainable service should mimic natural processes, and in so doing achieve energy efficiency, use future-oriented and life cycle perspectives, and evolve to adapt smoothly to changes in its environment. Moreover, it should incorporate sustain- ability into its supply chain and as its primary value and allow the customer to deliver sustainability to the next generation. Table 1 presents an example of quaternary that have to be considered when characterizing whether a service is sustainable.

\section{Examples}

\subsection{Car Wash}

A car wash is a daily process that is often provided by a distinct business as a service. First, it can be claimed that based on resource use, the most sustainable approach is not to clean your car. However, as maintaining a clean car has several incentives, the least of which is to preserve the car's value, it should be done using the most sustainable method. A distinctly unsustainable approach entails elements such as supplying the water through a constantly running pipe or employing children or other employees at unfair wages $(\Delta S>0)$. Alternatives, of course, exist, such as washing cars using the rag and bucket technique or using efficient, specialized car washing machines designed to minimize water usage $(\Delta \mathrm{S}$ $>0$ ). Taking it one step further, these machines often 
Table 1. Quaternary to characterize whether a service is based on nature's ground rules.

\begin{tabular}{ll}
\hline Rule & Questions \\
\hline \multirow{2}{*}{ Sustaining life-Future-oriented service } & 1. Does the service stand for a long time? \\
& 2. Does the service anticipate future scenarios? \\
& 3. Does the service use toxic materials? \\
& 1. Is the service necessary? \\
Energy-Service quantification & 2. Is it the most sustainable solution? \\
& 3. Does the service use minimum energy and materials? \\
& 5. Does the service lean on clean-technologies? \\
Entropy-Service quality & 1. Is it the most sustainable choice? \\
Life cycle-Service renewability & 2. Is the service broad, comprehensive and integrative? \\
& 3. What is the efficiency of the service compare to other alternatives? \\
Evolution-Spiral service & 1. How the service integrates with other services and manufacturing processes? \\
& 1. Does the service transfer sustainability over time? \\
& 2. Does the service learn and adapt to changes? \\
& 3. Does the customer supply sustainability to the next generations?
\end{tabular}

achieve their goal to reduce waste by implementing a system that recycles the water $(\Delta S \rightarrow 0)$, which makes a significant contribution to sustainability. Finally, in addition to considering water, a sustainable car wash must also strive to minimize its energy requirements, and the use of ecological detergents is ideal.

\subsection{Carbon Labeling}

One of the most useful and popular indicators of sustainability is the carbon footprint, which is a measure of the total greenhouse gas emissions from a process, or a product. Since each process involves the use of materials and energy that can be equivalently expressed via carbon dioxide emissions, the carbon footprint represents the total environmental impact of a process/product and enables between different processes/ products to be compared on a single scale. This important tool has gained wide acceptance, and in 2006, the Carbon Trust Company from the UK introduced a new service, named carbon labeling, to include in a product's label its carbon footprint just as, for example, calorie count or price is clearly stated.

Carbon labeling is a future-oriented service as it uses a simple, clear, and comparative tool to allow everyone designers, manufacturers, suppliers, and customers to participate in reducing human-generated greenhouse gas emissions to sustain the world. It has sustainability as a primary value, but it also integrates with other services and manufacturing and agricultural processes into a "super-service" that supplies sustainability as service by itself. The labeling of a tangible product with its carbon dioxide equivalent emission is a tool for producers and suppliers to evaluate the sustainability of their supply chain and determine whether cleaner technologies can be substituted while giving them an incentive to make the processes more sustainable and keep their carbon footprint in check by making a sustainable decision $(\Delta S>0)$.
To that end, carbon labeling gives the buyer the opportunity to choose between competing alternatives and make the most sustainable choice $(\Delta \mathrm{S} \rightarrow 0)$. Finally, carbon labeling allows each buyer to actively participate in promoting a more sustainable world, for example, by transferring sustainability to the next generation by using the label to choose the product with the smallest carbon footprint.

\section{REFERENCES}

[1] U. Karmakear, "Will You Survive the Service Evolution?" Harvard Business Review, Vol. 82, 2004, pp. 100-107.

[2] P. Maglio, S. Srinivasan, J. Kreulen and J. Spohrer, "Service Systems, Service Scientists, SSME, and Innovation," Communications of the ACM, Vol. 49, No. 7, 2006, pp. 81-85. doi:10.1145/1139922.

[3] R. Rust, "A Call for a Wider Range of Service Research," Journal of Service Research, Vol. 6, No. 3, 2004, p. 211. doi:10.1177/109467050463001

[4] R. C. Basole and W. B. Rouse, "Complexity of Service Value Networks: Conceptualization and Empirical Investigation," IBM Systems Journal, Vol. 47, No. 1, 2008, pp. 53-70. doi: $10.1147 / \mathrm{sj} .471 .0053$

[5] S. Alter, "Service System Fundamentals: Work System, Value Chain, and Life Cycle," IBM Systems Journal, Vol. 47, No. 1, 2008, pp. 71-85. doi:10.1147/sj.471.0071

[6] B. Hefley and W. Murphy, "Service Science, Management and Engineering, Education for the 21st Century," Springer, New York, 2008. doi:10.1007/978-0-387-76578-5

[7] J. A. Fitzsimmons, M. J. Fitzsimmons, "Service Management: Operations, Strategy, and Information Technology," 5th Edition, McGraw-Hill, Boston, 2006.

[8] A. W. Brown, M. Delbaere, P. Eales, S. Johnston and R. Weaver, "Realizing Service Oriented Solutions with the IBM Rational Software Development Platform," IBM 
Systems Journal, Vol. 44, No. 4, 2005, pp. 727-752. doi: $10.1147 / \mathrm{sj} .444 .0727$

[9] S. Dresner, "The Principles of Sustainability," 2nd Edition, EarthScan, Oxford, 2008.

[10] A. R. Edwards, "The Sustainability Revolution: Portrait of a Paradigm Shift," New Society Publishers, Gabriola Island, 2005.

[11] R. H. Crawford, "Validation of a Hybrid Life cycle Inventory Analysis Method," Journal of Environmental Management, Vol. 88, No. 3, 2008, pp. 496-506. doi:10.1016/j.jenvman.2007.03.024

[12] J. Guinée, "Handbook on Life Cycle Assessment: Operational Guide to the ISO Standards," Kluwer Academic Publishers, Dordrecht, 2002.

[13] H. Krcmar, "Informations Management," Springer, Berlin, 2010. doi:10.1007/978-3-642-04286-7
[14] M. Halme, G. Hrauda, C. Jasch, J. Kortman, H. Jonuschat, M. Scharp, D. Velte and P. Trindad, "Sustainable Consumer Services: Business Solutions for Household Market," EarthScan, Oxford, 2007.

[15] J. R. Galbraith, "Organizing to Deliver Solutions," Organizational Dynamics, Vol. 31, 2002, pp. 194-207.

[16] A. Wolfson, D. Tavor, S. Mark, M. Schermann and H. Krcmar, "S ${ }^{3}$-Sustainability and Services Science: Novel Perspective and Challenge," Service Science, Vol. 2, No. 4, 2010, pp. 216-224.

[17] C. D. Gretchen, "Nature's Services, Societal Dependence on Natural Ecosystems," Island Press, Washington, 1997.

[18] J. M. Smitha and H. C. Van Ness, "Introduction to Chemical Engineering Thermodynamics," 5th Edition, McGraw-Hill, New York, 1996. 